\title{
GI highlights from the literature
}

\author{
Mairi H McLean, Education editor
}

\section{BASIC SCIENCE \\ The therapeutic effect of metformin on type 2 diabetes depends on microbiota modulation}

- Wu H, Esteve E, Tremaroli V, et al. Metformin alters the gut microbiome of individuals with treatment-naive type 2 diabetes, contributing to the therapeutic effects of the drug. Nat Med 2017;23:850-58.

Metformin is widely used as a first-line medication for type 2 diabetes (T2D). Recent mouse models have shown that metformin increases the abundance of Akkermansia muciniphila (a strict anaerobe that exerts beneficial effects on glucose homeostasis), suggesting that its therapeutic effect may rely in the modulation of gut microbiota. In this double-blind randomised trial, patients with newly diagnosed T2D who were naive to treatment received either metformin $(n=22)$ or placebo $(n=18)$ for 4 months. In the metformin group, several alterations of microbiota were identified after the treatment including an increase of Escherichia and a decrease of Intestinibacter at the genus level. There was also an increase in $A$. muciniphila abundance and in the number of positive connections among microbes. Metformin directly promoted the growth of $A$. muciniphila in pure cultures. These findings were confirmed in a subgroup of patients in the placebo group who subsequently received metformin 6 months after the start of the study. Moreover, high-fatdiet-fed germ-free mice were transplanted with faecal samples from patients in the metformin group before and after metformin treatment, and only those who received faeces collected after 4 months of treatment experienced improvement of glucose tolerance. Finally, in two models of gut simulators, metformin was shown to influence, both in A. muciniphila and in Bilophila wadsworthia, the expression of several genes especially those encoding for metalloproteins or metal transporters. These results support the concept that some of the antidiabetic effects of metformin may depend on the modulation of the host microbiota.

\section{Fructose consumption and progressive NAFLD}

\section{Softic S, Gupta MK, Wang GX, et al. Divergent effects of glucose and fructose on hepatic lipogenesis and insulin signalling. J Clin Invest 2017;127:4059-74.}

Fructose is relatively uncommon in non-processed foods but is commonly used as a sweetener particularly in the form of high-fructose corn syrup in soft drinks. Population-level intake of fructose has increased over recent decades, and increased fructose consumption is associated with more severe liver disease in patients with non-alcoholic fatty liver disease (NAFLD). This paper demonstrates the mechanisms by which fructose consumption leads to liver injury in the context of obesity. High-throughput sequencing of RNA expression showed differing genetic expression profiles in mice given a high-fat diet (HFD) with water containing either glucose or fructose. HFD+glucose showed greatest effects on triglyceride synthesis and fatty acid oxidation, whereas HFD + fructose upregulated genes involved with fatty acid synthesis. Differences were also seen in gene expression of the insulin-signalling pathway where HFD+glucose upregulated the insulin receptor expression but HFD+fructose did not. Other data in the paper serve to confirm these findings: HFD+fructose caused a twofold increase in the cleaved, active form of SREBP1c, a critical regulator of lipogenesis, whereas glucose had little effect, and significantly more steatosis was seen in liver tissue from fructose-fed mice. Both liver and visceral adipose tissue mass were significantly higher in the HFD + fructose group. Interestingly,
HFD + glucose ameliorated insulin resistance in obese mice, whereas this was exacerbated in HFD + fructose, consistent with the observed upregulation of the insulin receptor by glucose. These data provide a biological explanation for the epidemiological observation of an association between fructose consumption and progressive NAFLD.

\section{The cellular source of Barrett's oesophagus}

- Jiang M, Li H, Zhang Y, et al. Transitional basal cells at the squamous-columnar junction generate Barrett's oesophagus. Nature 2017;550:529-33.

There are several theories on the cellular origins of Barrett's oesophagus. Jiang and colleagues suggest that cells at the gastro-oesophageal junction are the source of Barrett's as these exhibit properties of squamous and columnar differentiation, termed transitional or multilayered epithelium. The authors demonstrate that overexpression of Sox 2 (which has a role in maintaining pluripotency in embryonic stem cells) in cytokeratin $5+$ squamous cells results in hyperplasia that expands the transitional cell zone, lineage traced from the squamous cells $(p 63+)$ but not from the gastric cardia. This suggests that the squamous epithelium is the source of the transitional zone. They then show that this zone is maintained by a population of transitional progenitor cells (both p63 + and cytokeratin $7+$ (columnar)) and provide evidence for the presence of multipotent stem cells in this zone. When CDX2 expression is induced in cytokeratin $5+$ transitional zone progenitor cells, glands containing goblet cells and expressing intestinal lineage markers (AGR2, MUC2) appear. This suggests that intestinal metaplasia can be derived from transitional zone progenitor cells and is the first demonstration of metaplasia from cells located in the squamouscolumnar junction. On investigation of human Barrett's, the transitional zone appears to be expanded but no lineage tracing was presented. There are some questions around the translation of the murine data to the human disease due to anatomical differences. Nevertheless, this is an interesting model that shows some cells are more susceptible to producing intestinal cells in the upper gastrointestinal tract and is the first study to show glandular differentiation from a non-glandular source.

\section{CLINICAL PRACTICE}

\section{Reactive endoscopy - the endoscopist as the allergist?}

- Akkerdaas J, van Esch BCAM, van Ree R, et al. Abnormal responses to local esophageal food allergen injections in adult patients with eosinophilic esophagitis. Gastroenterology 2017. doi: 10.1053/j.gastro.2017.08.062. [Epub ahead of print 01 Sep 2017].

Eosinophilic oesophagitis (EoE) is reaching a prevalence similar to that of inflammatory bowel disease. Although the aetiology remains controversial especially in view of protein pump inhibitor (PPI) responsive disease, there has been great success with elimination diets based on the hypothesis that at least some patients with EoE have topical oesophageal sensitivity to food allergens. Citing the lack of sensitivity and specificity of skin and blood allergy testing, the authors describe the injection of six allergens injected into the oesophaguses (EPT) of eight patients as well as three control patients. The patients underwent a further endoscopy after 24 hours so that delayed responses could be detected. Biopsies were also taken and skin and blood allergy testing was also carried out on the same day. Five of the treatment patients developed an acute reaction with a further two developing a delayed reaction. Interestingly, the skin and blood allergy testing correlated with EPT in only three of the eight 
patients. This pilot study has an interesting potential. If EPT isolates the EoE trigger allergen more reliably than skin or blood testing, then there is a new potential for endoscopic allergen testing with perhaps more emphasis on targeted rather than empirical elimination diets.

\section{Roux-en-Y gastric bypass provides durable weight loss and effective remission from metabolic morbidity}

Adams TD, Davidson LE, Litwin, SE, et al. Weight and metabolic outcomes 12 years after gastric bypass. N Engl J Med 2017;377:1143-55.

A number of short-term studies have shown the efficacy of several bariatric surgical procedures in reversing metabolic conditions associated with obesity, but to date there has been a paucity of prospectively collected long-term follow-up data showing the durability of this effect. Adams and colleagues report outcome data from a prospectively recruited cohort of 835 severely obese patients attending a single centre in Utah, USA, and followed up for 12 years. A total of 418 patients underwent Roux-en-Y gastric bypass, and 417 did not receive surgery. Follow-up data were available from 752 individuals and included percentage original weight lost, incidence and remission rates for type 2 diabetes, dyslipidaemia and hypertension. Among patients who underwent surgery, sustained weight loss of $35.0 \mathrm{~kg}$ (95\% CI 38.4 to 31.7 ; mean per cent change, $26.9 \%$ ) was observed. For patients who did not undergo surgery, durable weight loss was not observed (mean weight loss $2.9 \mathrm{~kg}(6.9$ to -1.0 ; mean per cent change, $2.0, \mathrm{P}<0.01)$. The incidence of type 2 diabetes was lower in the surgical group (OR 0.08 (0.03 to $0.24) ; \mathrm{P}<0.001)$, and remission of type 2 diabetes was observed in $51 \%$ of affected individuals treated with surgery (OR 8.9 (2.0 to 40.0); $\mathrm{P}<0.001)$. Similar improvements for incidence and remission of hypertension and dyslipidaemia in the surgical group are also reported. This article demonstrates that Roux-en-Y gastric bypass can maintain durable weight loss and sustain remission from associated metabolic morbidity. Similar data are needed for other common bariatric surgery techniques to enable rational comparison of these procedures.

\section{The link between treatment of HCV with DAAs and HCC: time to draw the line?}

Kanwal F, Kramer J, Asch SM, et al. Risk of hepatocellular cancer in HCV patients treated with direct-acting antiviral agents. Gastroenterology 2017;153:996-1005.

There is controversy on the risks of hepatocellular carcinoma (HCC) in hepatitis $\mathrm{C}$ virus (HCV) positive patients who undergo treatment with direct-acting antiviral agents (DAAs). Some studies suggest treatment is associated with a higher incidence and altered behaviour of cancer. The widespread use of these agents in both cirrhotic and non-cirrhotic subjects makes it difficult to assess the risk of the agents per se versus the risk incurred by the stage of liver disease. This retrospective cohort study, involving 25232 patients with HCV who were treated with DAA, hoped to address this question. A total of 19518 patients had sustained viral response (SVR) and 2982 did not achieve SVR with a clinical follow-up of 20415 and 2547 patient-years, respectively. The incidence rate of HCC was 0.90 (95\% CI 0.77 to 1.03 ) and 3.45 (95\% CI 2.73 to 4.18$)$, respectively, with a HR of $0.28(0.22-0.36)(\mathrm{P}<0.001)$. Patients with cirrhosis had higher annual incidence of HCC after SVR (1.82 vs $0.34 / 100$ person-years in patients without cirrhosis; adjusted HR 4.73, 95\% CI 3.34 to 6.68). This large study conforms to very well-known dogmas from the interferon therapy era, namely, that cirrhosis enhances the risk of HCC even after clearance of the virus and patients with SVR had a lower risk of HCC. The impact of this data is that continued surveillance of all patients with HCV cirrhosis should be the accepted standard, even if clearance of the virus is achieved. The evidence that DAAs promote HCC is most likely a consequence of underpowered studies in the past.

\section{REVIEWERS}

Dr Gianluca Ianiro, Dr Richard Parker, Dr Stuart McDonald, Dr Sebastian Zeki, Dr Michael Burkitt, Dr Ashis Mukhopadhya.

\section{JOURNALS REVIEWED}

Nature Medicine, Journal of Clinical investigation, Nature, Gastroenterology, New England Journal of Medicine.

Competing interests None declared.

Provenance and peer review Not commissioned; internally peer reviewed.

(c) Article author(s) (or their employer(s) unless otherwise stated in the text of the article) 2018. All rights reserved. No commercial use is permitted unless otherwise expressly granted.

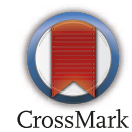

To cite McLean MH. Gut 2018:67:194-195. doi:10.1136/gutjnl-2017-315602 